\title{
Coordinating Electric Vehicle Flow Distribution and Charger Allocation by Joint Optimization
}

\begin{abstract}
A two-stage stochastic programming model is established to minimize EV's expected total journey time under stochastic traffic conditions, by jointly optimizing the allocation of chargers and the distribution of $\mathrm{EV}$ flows. Based on sample average approximation, a feasible deterministic equivalent of the original stochastic problem is obtained. Then, a hybrid solution method, composing of a Tabu-based search and sequential quadratic programming (SQP), is proposed. The Tabu heuristic manages the charger allocation problem, where each solution candidate undergoes a second-stage EV flow optimization. SQP is applied to optimially distribute the $\mathrm{EV}$ flows, which is proved to be a convex problem. Extensive simulations are carried out using the eastern Massachusetts highway network. Results show that the proposed algorithm outperforms existing approaches by finding high-quality charger allocation and optimal flow distribution policies under different traffic conditions. Additionally, the two-stage model designates charging resource sufficiency by statistically estimating a lower bound for the number of chargers to allocate, which in practice helps to prevent over-investment on charging resources.
\end{abstract}

Index Terms-Electric vehicle (EV), traffic flow distribution, charger allocation, two-stage stochastic programming, convex optimization.

\section{INTRODUCTION}

A CCORDING to the "Global Electric Vehicle (EV) Outlook 2019" published by International Energy Agency [1], the projected EV fleet under the EV30@30 campaign [2], which aims for at least $30 \%$ new EV sales by 2030 , has a global well-to-wheel (WTW) greenhouse gas (GHG) emission of less than 230 million tonnes of carbon-dioxide equivalent (Mt CO2-eq). By contrast, the equivalent internal combustion engine (ICE) vehicle fleet would emit an enormous $770 \mathrm{Mt}$ $\mathrm{CO} 2$-eq. Such GHG emission reduction brought by transport electrification can potentially be even more significant, given a faster decarbonization of electricity generation using renewable energy.

Although the scale of electric mobility has been expanding rapidly (exceeding 5.1 million globally in 2018), there is still a long way to go towards the EV30@30 target. As for the prospective drivers of $\mathrm{EV}$, what they care most about is the EV driving experience. Unfortunately, EV's limited driving range and long re-charge time can result in the so-called "range anxiety", indicating the EV drivers' concern of running out of power before arriving at the destinations.

To alleviate the range anxiety among EV drivers and consequently promote a large-scale adoption of EVs, extensive research efforts have been made in recent years, focusing on the planning of EV's en-route recharge activities [3], [4], [5], [6], [7], [8], [9], [10] and the design of charging infrastructure [11], [12], [13], [14], [15], [16], [17], [18]. For instance, [7] suggests efficient itineraries under timedependent stochastic traffic condition for EVs used in logistics industry; [8] considers a city-wide system that recommends the location and occasion of re-charge for plug-in electric taxi by coordinating with the charging infrastructure via an information center; [16] proposes to place charging stations, by capturing the competitive and strategic re-charge behaviors among EV drivers using game theory; in [17], the charging infrastructure and the coupled power distribution systems are planned collaboratively to not only minimize the joint cost but also maximize the utilization.

Despite that the aforementioned works have covered many practical conditions and constraints in their models, the planning of EV's en-route re-charge activities and the allocation of charging resources were addressed independently. On one hand, charging resources are allocated according to some patterns of EV's re-charge activities which are usually generated based on existing data sets [15], [16] or models [17], [18]. On the other hand, the EV's en-route re-charge activities are planned with the support of specified charging infrastructure which may be designed by real-world data [7], [10] or random assignment [8], [9]. There is no doubt that these two problems are indeed closely related. An obvious issue of making independent decisions is that any changes in EV's re-charging pattern or charging infrastructure can potentially lead to a different result. It might be feasible to adjust the planning strategy for EV's en-route re-charging according to the actual condition, e.g. available charging resources, but not the other way around, because the deployment of charging infrastructure is more of an irreversible process.

Therefore, to fully empower an electrified transportation system, it is essential to coordinate the present deployment of charging infrastructure and subsequent planning of EV's re-charge activities, which is also the focus of this work. The main contributions are summarized as follows:

1) A two-stage stochastic programming model is defined over a road network with stochastic traffic flow to characterize the coordination between EV flow distribution and charger allocation. The two-stage decisions can then jointly minimize EV's expected total journey time to improve the driving experience.

2) A feasible deterministic equivalent of the original joint optimization problem is derived based on sample average approximation (SAA). The quality of the SAA estimator is evaluated by estimating the objective function bounds of the original problem.

3) The EV flow distribution problem is proved to be convex, and can thus be solved optimally and efficiently using sequential quadratic programming (SQP). Since the optimal solution becomes available, it outperforms other existing works, for example the heuristic methods, as used in [3]. 
4) A hybrid approach, incorporating a dedicated Tabubased heuristic and SQP, is designed to solve the joint optimization problem. It not only outperforms existing ones by yielding a high-quality charger allocation and optimal EV flow distribution policies under different traffic conditions, but also facilitates the estimation of a lower bound for the number of chargers to allocate. In practice, such a lower bound helps to prevent the overallocation of charging resources.

The rest of the paper is organized as follows. The two-stage stochastic programming problem is formulated in Sect. II, while the design of solution algorithms is introduced in Sect. III. In Sect. IV, simulation results for an illustrative example of the eastern Massachusetts highway network and discussions are presented. Finally, conclusions are given in Sect. V.

\section{System Model And Problem Formulation}

\section{A. Road Network with Stochastic Traffic Flow}

A road network with charging infrastructure installed can be represented by a directed and weighted graph $\mathcal{G}=(\mathcal{V}, \mathcal{E}, \mathcal{A})$. $\mathcal{V}$ is the set of nodes (points of interest) including charging station locations denoted by $\mathcal{V}_{c} \subset \mathcal{V} . \mathcal{E}=\{(i, j) \mid i, j \in \mathcal{V}, i \neq$ $j\}$ is the set of edges and $\mathcal{A}=\left\{\left(d_{i j}, \tau_{i j}^{0}, q_{i j}, \delta_{i j}\right) \mid(i, j) \in \mathcal{E}\right\}$ is the set of weights associated to the edges such that

- $d_{i j}$ is the traveling length of the edge $(i, j)$ (in $\mathrm{km}$ );

- $\tau_{i j}^{0}$ denotes the free-flow traveling time of the edge $(i, j)$ (in hours);

- $q_{i j}$ stands for $(i, j)$ flow capacity (in vehicles per hour);

- $\delta_{i j}$ is the proportion of $q_{i j}$ being occupied by non-EVs (e.g. ICE vehicles) and those EVs that demand no enroute re-charging.

Here, $\delta_{i j}$ is assumed as a realization of a random variable $\Delta_{i j}$ which follows a normal distribution, i.e., $\Delta_{i j} \sim$ $\mathcal{N}\left(\mu_{i j}, \sigma_{i j}^{2}\right)$. Based on the widely used Bureau of Public Roads (BPR) volume-delay function [19], the actual traveling time from $i$ to $j$ can be estimated by

$$
\tau_{i j}=\tau_{i j}^{0}\left(1+\alpha\left[\delta_{i j}+\frac{\lambda_{i j}}{q_{i j}}\right]^{\beta}\right)
$$

where $\lambda_{i j}$ (in vehicles per hour) denotes the rate of EV flow at edge $(i, j)$ that demands en-route re-charging; $\alpha$ and $\beta$ are tuning parameters that control how traffic congestion affects the traveling time. Due to the existence of uncertainty $\delta_{i j}$, the traveling time $\tau_{i j}$ characterized by (1) is essentially stochastic and should be taken into consideration when planning EV's en-route re-charge activities, i.e. $\lambda_{i j}$.

\section{B. Distribution of EV Flows}

En-route re-charging is necessary for EVs traveling from a source node $m$ to a destination node $n$ in the road network $\mathcal{G}$, if their driving range $d_{r}<d(m, n)$ where $d(m, n)$ denotes the shortest path length from $m$ to $n$. This source-destination pair, denoted by $(m \mapsto n)$, is said to be unreachable to the EVs. In this case, the EVs are to be re-distributed to a set of charging stations $\mathcal{V}_{s} \subseteq \mathcal{V}_{c}$, which provide re-charging services. Assuming the EV flow departing from node $m$ (to $n$ ) follows a Poisson process with an intensity $\lambda$, such redistribution of EV flow is essentially splitting the Poisson process to multiple Poisson processes [20]. An illustrative example is given in Fig. 1. With the re-charging services available at $\mathcal{V}_{s}=\left\{s_{1}, s_{2}, \cdots, s_{6}\right\}$, the EV flow at rate $\lambda$ can be distributed onto the eligible paths from $m$ to $n$. For example, $m \Rightarrow s_{1} \Rightarrow n$ is an eligible path. All the eligible paths then form a tree structure $T_{m n}$, where the source $m$ acts as the root and destination $n$ is excluded. The charging stations $s_{1}, s_{4}, s_{5}, s_{6}$ are the leaf nodes from which EVs can arrive $n$ without further re-charging. It is remarked that two successive charging stations (e.g. $s_{2}$ and $s_{4}$ ) may not be directly connected, in which case EVs travel the shortest path in between.

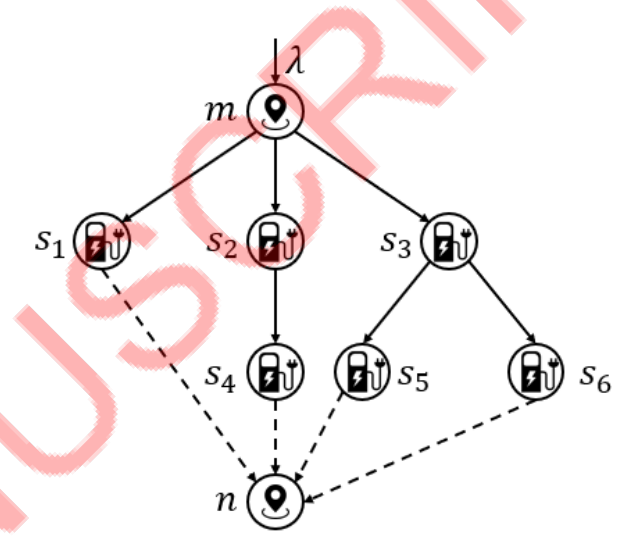

Figure 1: Illustration of the EV flow's distribution due to enroute re-charging.

For the sake of demonstration, whilst reflecting the practical situation, the following assumptions are made for the charging stations $\mathcal{V}_{c}$ in this work.

- All of the chargers installed at $\mathcal{V}_{c}$ are level-3 [21] and with the same specifications, e.g. 50KW DC.

- A charging station provides charging services under the first-in first-out principle.

- The charging time with a single charger is characterized by a general distribution $F_{c}$, e.g. truncated normal distribution, with mean $\frac{1}{\mu_{c}}$ and variance $\sigma_{c}^{2}$.

Since the EV arrivals at charging stations are Markovian, an individual EV's average delay (including both queuing and charging times) at a charging station $s_{k} \in \mathcal{V}_{c}$ with $c_{k}$ chargers can be approximated by $M / G / K$ queuing model [22] with $K=c_{k}$, which is given by

$$
\mathbb{E}\left[W^{M / G / c_{k}}\left(s_{k}\right)\right]=\frac{C V^{2}+1}{2} \mathbb{E}\left[W^{M / M / c_{k}}\left(s_{k}\right)\right]
$$

where $C V$ is $F_{c}$ 's coefficient of variation; $\mathbb{E}\left[W^{M / M / c_{k}}\left(s_{k}\right)\right]$ refers to the EV's average delay given exponentially distributed charging time with a parameter of $\mu_{c}$ such that

$$
\mathbb{E}\left[W^{M / M / c_{k}}\left(s_{k}\right)\right]=\frac{B\left[\lambda\left(s_{k}\right)\right]}{c_{k} \mu_{c}-\lambda\left(s_{k}\right)}+\frac{1}{\mu_{c}}
$$

with $B\left[\lambda\left(s_{k}\right)\right]$ being the Erlang $\mathrm{C}$ formula and $\lambda\left(s_{k}\right)$ being the aggregated rate of EV flows arriving at $s_{k}$. 


\section{Joint Optimization of EV Flow Distribution and Charger Allocation}

Expanding the charging infrastructure is one of the most direct initiatives to alleviate EV drivers' range anxiety. Obviously, how to allocate chargers at the charging stations largely affects how well the EV flows can be distributed. Therefore, these two decisions have to be made coordinately.

Here, a joint optimization problem is considered to characterize such a coordination. A given number of chargers is firstly to be allocated to the charging stations. Then, EV flows are needed to be distributed onto the road network according to the realizations of traffic condition uncertainties, i.e. $\delta_{i j}$. This formulation naturally results in a two-stage stochastic programming problem, which has also been considered in many other practical applications [23]. However, it should be emphasized that the formulation and solution algorithm are dedicated to the problem in concern.

In this work, the joint optimization problem aims to minimize the EV's expected total journey time. Mathematically, it is formulated as follows

$$
\begin{array}{ll}
\min _{\boldsymbol{c}^{\prime}} & \mathbb{E}\left[H^{*}(\boldsymbol{c}, \boldsymbol{\delta})\right] \\
\text { s.t. } & \sum_{k=1}^{\left|\mathcal{V}_{c}\right|} c_{k}^{\prime}=U \\
& \boldsymbol{c}=\boldsymbol{c}^{\prime}+\boldsymbol{c}^{\mathbf{0}}
\end{array}
$$

where $|\cdot|$ is the cardinality of a set; $\boldsymbol{c}^{\prime}=\left\{c_{k}^{\prime}\right\} \in \mathbb{Z}^{\left|\mathcal{V}_{c}\right|}$ is the first-stage decision vector indicating the number of chargers to be further allocated at each of charging stations and $c_{k}^{\prime} \geq 0$; $U$ is the total number of chargers to be installed; $\boldsymbol{c}^{\mathbf{0}}=\left\{c_{k}^{0}\right\} \in$ $\mathbb{Z}^{\left|\mathcal{V}_{c}\right|}$ are the numbers of chargers originally installed and $c_{k}^{0} \geq$ $0 ; \boldsymbol{\delta} \in \mathbb{R}^{|\mathcal{E}|}$ is a vector of $\delta_{i j}$ realization; $H^{*}(\boldsymbol{c}, \boldsymbol{\delta})$ indicates the optimal value of second-stage EV flow distribution problem, which is to be further explained in the next sub-section.

\section{Formulation of EV Flow Distribution Problem}

Given the charger allocation $c$ and traffic condition $\delta$, the second-stage optimization aims to minimize EV's expected delay $H(\boldsymbol{c}, \boldsymbol{\delta})$, which includes not only the traveling delay, but also the en-route re-charge delay. To tackle this optimization problem, we can first identify $\mathcal{V}_{s}$ and extract the tree $T_{m n}$ for each unreachable source-destination pair $(m \mapsto n)$. This can be solved by the Iterative Tree Construction Algorithm (ITCA) as described in [3], [24], which is essentially a direct heuristic algorithm, and thus details are omitted here.

The EV flow at rate $\lambda(m, n)$ for $(m \mapsto n)$ is then distributed to the eligible paths in $T_{m n}$. Let $\lambda_{i j}^{(m n)}$ denote the portion of $\lambda(m, n)$ assigned to edge $(i, j)$, the problem of EV flow distribution under stochastic traffic conditions can be formulated as

$$
\min _{\Lambda} H(\boldsymbol{c}, \boldsymbol{\delta})=\frac{1}{N} \sum_{\substack{m, n \in \mathcal{V}, m \neq n ; \\ d_{r}<d(m, n)}}\left\{\frac{1}{\lambda(m, n)} \sum_{P \subseteq T_{m n}} \lambda_{P}^{(m n)} \tau_{P}\right\}
$$

subject to:

$$
\begin{aligned}
& \sum_{u=C(m)} \lambda_{m u}^{(m n)}=\lambda(m, n), \\
& \lambda_{i j}^{(m n)}=\sum_{u=C(j)} \lambda_{j u}^{(m n)}, \quad \forall j \in T_{m n} \backslash\left\{m \cup L\left(T_{m n}\right)\right\} \\
& \lambda_{i j}^{(m n)} \geq 0, \\
& \lambda_{i j}=\sum_{\substack{m, n \in \mathcal{V}, m \neq n ; \\
d_{r}<d(m, n)}} \lambda_{i j}^{(m n)}, \quad \forall(i, j) \in \mathcal{E} \\
& \lambda\left(s_{k}\right)=\sum_{\substack{(i, j) \in \mathcal{E}, j=s_{k} \\
\lambda\left(s_{k}\right)<c_{k} \mu_{c}, \quad \forall s_{k} \in \mathcal{V}_{s}}} \lambda_{i j}, \quad \forall s_{k} \in \mathcal{V}_{s} \\
& \text { (m) }
\end{aligned}
$$

where $\boldsymbol{\Lambda}=\left\{\lambda_{i j}^{(m n)}\right\} \in \mathbb{R}^{D}$ and $D=\sum_{m, n}\left|B R\left(T_{m n}\right)\right|$ with $B R\left(T_{m n}\right)$ denoting the set of branches in $T_{m n} ; N$ is the total number of trees which is also equal to the number of unreachable source-destination pairs; $C(j)$ indicates the set of children nodes of node $j ; P \subseteq T_{m n}$ refers to an eligible path in $T_{m n}$ associated with an EV flow of rate $\lambda_{P}^{(m n)}\left(=\lambda_{L(P), n}^{(m n)}\right)$; $\tau_{P}$ is the total delay for an EV to travel from $m$ to $n$ via path $P$ which is given by

$$
\tau_{P}=\tau_{L(P), n}+\sum_{(i, j) \in P} \tau_{i j}+\sum_{s_{k} \in P} \mathbb{E}\left[W^{M / G / c_{k}}\left(s_{k}\right)\right]
$$

where $L(P)$ indicates the node just before the destination $n$ in path $P ; \tau_{L(P), n}$ and $\tau_{i j}$ evaluate the time delay to travel corresponding path segments using (1) based on $\lambda_{i j}$ and $\delta_{i j}$, respectively.

As for the constraints, (8) and (9) require that the inflow rate equals to the summation of out-flow rates at any $s_{k}$ in the tree $T_{m n} ;(10)$ specifies the non-negativity of the decision variables; (11) indicates that the EV flow at edge $(i, j)$ essentially contains all the EVs that travel for any unreachable pair via $(i, j) ;(12)$ defines the aggregated EV flow rate $\lambda\left(s_{k}\right)$ arriving at charging station $s_{k}$ and (13) is to avoid overutilization of any charging station $s_{k} \in \mathcal{V}_{s}$.

\section{E. A Feasible SAA for Joint Optimization Problem}

Since problem (7) is defined over a network with stochastic traffic flow, the joint problem (4) cannot be solved directly. To address this issue, SAA method as suggested in [23] is adopted to approximate $\mathbb{E}\left[H^{*}(\boldsymbol{c}, \boldsymbol{\delta})\right]$ using randomly generated samples of vector $\delta$, i.e. $\left[\delta^{1}, \delta^{2}, \cdots, \delta^{I}\right]$, by the average

$$
\hat{q}_{I}(\boldsymbol{c})=I^{-1} \sum_{l=1}^{I} H^{*}\left(\boldsymbol{c}, \boldsymbol{\delta}^{l}\right)
$$

Then, (4) can be transformed to a deterministic equivalent, which is a collection of minimization problems given by

$$
\begin{array}{ll}
\min _{\boldsymbol{c}^{\prime}} & \hat{q}_{I}(\boldsymbol{c}) \\
\text { s.t. } & \sum_{k=1}^{\left|\mathcal{V}_{c}\right|} c_{k}^{\prime}=U
\end{array}
$$




$$
c=c^{\prime}+c^{0}
$$

The constraint (13), i.e. $\lambda\left(s_{k}\right)<c_{k} \mu_{c}, \forall s_{k} \in \mathcal{V}_{s}$, is a strict inequality, which leads to an open feasible domain. To ensure the existence of an optimal solution, it can be relaxed by introducing an additional parameter $\epsilon$ which controls the percentage of charging resources being retained for the EVs that improvises for re-charging. Therefore, (13) is modified as

$$
\frac{\lambda\left(s_{k}\right)}{c_{k} \mu_{c}} \leq 1-\epsilon, \quad \forall s_{k} \in \mathcal{V}_{s}
$$

Note that the requirement of sufficient charging capacity for the routing options in ITCA needs to consider $\epsilon$ accordingly as well.

\section{Solution Algorithms}

\section{A. Convexity of EV Flow Distribution Problem}

An optimization problem can be efficiently solved if it can be recognized or transformed into a convex one [25]. Hence, before proposing the algorithm for solving the optimization problem (16), the following useful theorem is firstly established:

Theorem 1. The EV flow distribution problem (7) is convex.

Proof. Combining (11) and (12), the EV's aggregated arrival rate at charging station $s_{k}, \lambda\left(s_{k}\right)$, is related to the decision variables $\lambda_{i j}^{(m n)}$ as follows

$$
\lambda\left(s_{k}\right)=\sum_{\substack{(i, j) \in \mathcal{E}, j=s_{k}}} \sum_{\substack{m, n \in \mathcal{V}, m \neq n ; \\ d_{r}<d(m, n)}} \lambda_{i j}^{(m n)}, \quad \forall s_{k} \in \mathcal{V}_{s}
$$

Substituting (20) into (19), one has

$$
\left[\sum_{\substack{(i, j) \in \mathcal{E}, j=s_{k}}} \sum_{\substack{m, n \in \mathcal{V}, m \neq n ; \\ d_{r}<d(m, n)}}\left(\lambda_{i j}^{(m n)}\right)\right]-c_{k} \mu_{k}(1-\epsilon) \leq 0
$$

The left-hand side of inequality (21) for each $s_{k}$ is essentially a linear function (thus convex) with respect to $\lambda_{i j}^{(m n)}$.

On the other hand, since $\lambda(m, n)$ is basically split over the branches of $T_{m n}$ (see (8) and (9)), for any $\lambda_{i j}^{(m n)}$, it is always true that

$$
\lambda_{i j}^{(m n)}+\sum_{\exists(a, b) \subseteq T_{m n}} \lambda_{a b}^{(m n)}-\lambda(m, n)=0
$$

where $\lambda_{a b}^{(m n)}$ are the EV flows assigned at some branches $(a, b)$ in $T_{m n}$. The left-hand side of the equality is an affine function with a translation of $-\lambda(m, n)$. Therefore, it can be concluded that the feasible region of problem (7) is convex [25].

It remains to show that the objective $H(\boldsymbol{c}, \boldsymbol{\delta})$ is a convex function of $\boldsymbol{\Lambda}$. It is noted that $H(\boldsymbol{c}, \boldsymbol{\delta})$ is inherently a weighted aggregation of a collection of $\tau_{P}$ as defined in (14). Therefore, proving the convexity of (14) would complete the proof, as a non-negative weighted sum of convex functions remains convex. Referring to (14), the first two terms and the third term are essentially (1) and (2), which can be proved as convex functions by the following.
1) It is observed that the rate of EV flow at edge $(i, j), \lambda_{i j}$, is a linear aggregation of the entries of $\boldsymbol{\Lambda}$. (see (11)). With $\Lambda \in \mathbb{R}^{D}$, (1) essentially defines a function $\tau_{i j}$ : $\mathbb{R}^{D} \rightarrow \mathbb{R}$, for each $(i, j) \in \mathcal{E}$. To prove the convexity of $\tau_{i j}$, its Hessian, Hess $\tau_{i j}$, on $\mathbb{R}^{D}$ needs to be at least positive semi-definite.

An entry of Hess $\tau_{i j}$ can be computed by

$$
\begin{aligned}
\left(\operatorname{Hess} \tau_{i j}\right)_{s t} & =\frac{\partial^{2} \tau_{i j}}{\partial \lambda_{s} \partial \lambda_{t}} \\
& =\frac{\partial^{2} \tau_{i j}}{\partial \lambda_{i j}^{2}} \cdot \frac{\partial \lambda_{i j}}{\lambda_{s}} \cdot \frac{\partial \lambda_{i j}}{\lambda_{t}}+\frac{\partial \tau_{i j}}{\partial \lambda_{i j}} \cdot \frac{\partial^{2} \lambda_{i j}}{\partial \lambda_{s} \partial \lambda_{t}}
\end{aligned}
$$

where $\lambda_{s}$ and $\lambda_{t}$ are the entries of $\boldsymbol{\Lambda}$. Therefore, for the diagonal terms of Hess $\tau_{i j}$, if $\lambda_{s}$ is aggregated by $\lambda_{i j}^{(m n)}$, one has $\left(\operatorname{Hess} \tau_{i j}\right)_{s s}=\frac{\partial^{2} \tau_{i j}}{\partial \lambda_{i}^{2}}$; otherwise, $\left(\operatorname{Hess} \tau_{i j}\right)_{s s}=$ 0 . For off-diagonal terms, there are three cases:

- If both $\lambda_{s}$ and $\lambda_{t}$ are not aggregated by $\lambda_{i j}^{(m n)}$, then $\left(\text { Hess } \tau_{i j}\right)_{s t}=0$

- If only one of them is aggregated, $\left(\operatorname{Hess} \tau_{i j}\right)_{s t}=0$

- If both of $\lambda_{s}$ and $\lambda_{t}$ are aggregated, $\left(\operatorname{Hess} \tau_{i j}\right)_{s t}=$ $\left(\text { Hess } \tau_{i j}\right)_{t s}=\frac{\partial^{2} \tau_{i j}}{\partial \lambda_{i j}^{2}}$.

Since the non-zero entries in Hess $\tau_{i j}$ are essentially identical and equals to $\frac{\partial^{2} \tau_{i j}}{\partial \lambda_{i j}^{2}}$, a succession of elementary operations can be performed and Hess $\tau_{i j}$ becomes

$$
\operatorname{Hess} \tau_{i j}=\frac{\partial^{2} \tau_{i j}}{\partial \lambda_{i j}^{2}}\left[\begin{array}{cc}
\mathbf{1}_{\eta \times \eta} & \mathbf{0}_{\eta \times(D-\eta)} \\
\mathbf{0}_{(D-\eta) \times \eta} & \mathbf{0}_{(D-\eta) \times(D-\eta)}
\end{array}\right] .
$$

where 1 and 0 represent the matrices of one and zero with corresponding sizes, respectively; $\eta$ denotes the total number of non-zero entries in Hess $\tau_{i j}$. Moreover, $\frac{\partial^{2} \tau_{i j}}{\partial \lambda_{i j}^{2}}$ can be derived as

$$
\frac{\partial^{2} \tau_{i j}}{\partial \lambda_{i j}^{2}}=\frac{\tau_{i j}^{0} \alpha \beta(\beta-1)}{q_{i j}^{2}}\left(\delta_{i j}+\frac{\lambda_{i j}}{q_{i j}}\right)^{\beta-2}
$$

By considering the typical BPR formula, i.e. $\alpha=0.5$ and $\beta=4$ [19], it can be easily deduced that (25) is positive.

Since the eigenvalues of $\mathbf{1}_{\eta \times \eta}$ equal to $\eta$ and 0 , with multiplicity of 1 and $\eta-1$, respectively [26], Hess $\tau_{i j}$ should also have a single positive eigenvalue (others are all zero) according to the Sylvester's law of inertia [26]. As a result, the Hessian of (1) with respect to $\boldsymbol{\Lambda}$ is positive semi-finite.

2) As implied by (20), the entries of $\boldsymbol{\Lambda}$ are also linearly aggregated for $\lambda\left(s_{k}\right)$, and thus the convexity proof for (1) is also applicable here. Similarly, to show that the Hessian of $\mathbb{E}\left[W^{M / G / c_{k}}\left(s_{k}\right)\right]$ on $\mathbb{R}^{D}$ is also positive semidefinite, the second derivative of $\mathbb{E}\left[W^{M / G / c_{k}}\left(s_{k}\right)\right]$ with respect to $\lambda\left(s_{k}\right)$ needs to be positive. In [27], (3) has been proved to be a convex function of arrival rate on $\mathbb{R}$. The approximation (2) is thus also convex because the scalar, i.e. $\frac{C V^{2}+1}{2}$, is always positive, which completes the proof. 


\section{B. Optimization of EV Flow Distribution}

Since problem (7) is convex (according to Theorem 1), and also strictly feasible, i.e. Slater's constraint qualification holds, there exist dual optimal $\psi^{*}$ and $\omega^{*}$, together with the optimal EV flow rates $\boldsymbol{\Lambda}^{*}$, satisfying the Karush-Kuhn-Tucker (KKT) conditions. Classical convex programming tools can be applied to obtain $\Lambda^{*}$ efficiently [25] and SQP method [28] is adopted here. It is remarked that problem (7) can only be solved given a sample of vector $\delta$, i.e. stochastic traffic flow. The approximation of $\mathbb{E}\left[H^{*}(\boldsymbol{c}, \boldsymbol{\delta})\right]$ using SAA method essentially yields a collection of EV flow distribution policies with respect to different traffic conditions under charger allocation $c$.

\section{A Hybrid Approach for Feasible SAA}

A hybrid approach, which incorporates SQP into a Tabubased [29] charger allocation algorithm (CAA), is designed to solve problem (16). The mechanisms of CAA are detailed as follows.

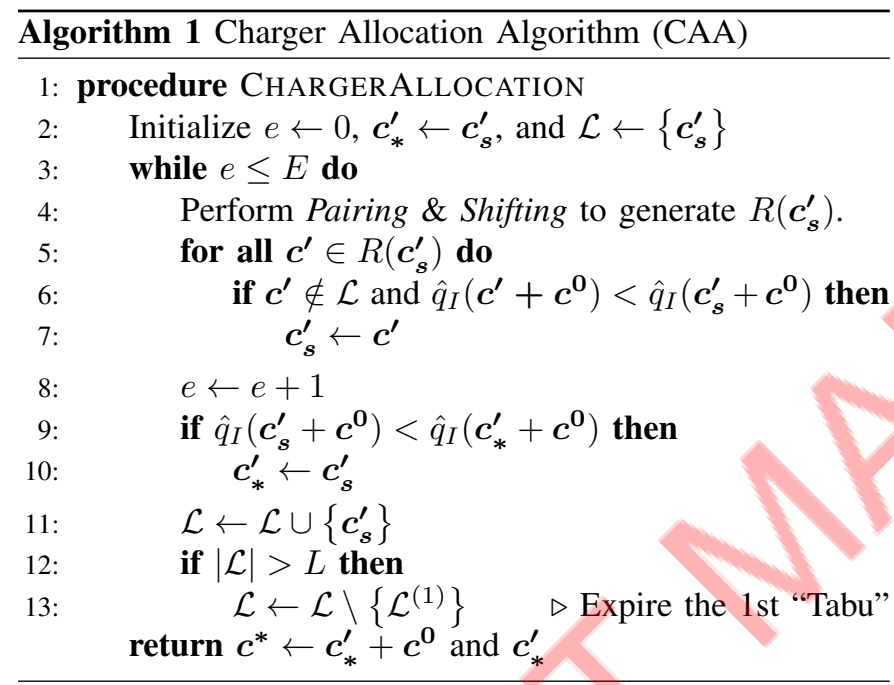

Note: The optimal EV flow distribution solution $\hat{q}_{I}(\cdot)$ for each candidate of charger allocation is obtained by SQP; $c^{0}$ are the numbers of chargers originally installed.

1) Encoding Method: The solution candidate is simply $\boldsymbol{c}^{\prime}=\left\{c_{k}^{\prime}\right\} \in \mathbb{Z}^{\left|\mathcal{V}_{c}\right|}$, where $c_{k}^{\prime}$ implies the number of chargers to be assigned at the charging station $s_{k}$.

2) Neighborhood Structure: The basic idea of Tabu search is to explore the solution space by seeking improved solution among the neighbors of $\boldsymbol{c}^{\prime}$. Therefore, a two-step neighborhood structure that generates feasible neighbors of $\boldsymbol{c}^{\prime}$ is devised.

a) Pairing: all the elements of $\boldsymbol{c}^{\prime}$ are randomly paired;

b) Shifting: for each element pair $c_{i}^{\prime}$ and $c_{j}^{\prime}$, if $c_{i}^{\prime}, c_{j}^{\prime} \neq$ 0 , then one of them, say $c_{i}^{\prime}$, will shift $c_{i}^{t} \in\left[1, c_{i}^{\prime}\right]$ chargers to $c_{j}^{\prime}$. Consequently, one has $c_{i}^{\prime} \leftarrow c_{i}^{\prime}-c_{i}^{t}$, and $c_{j}^{\prime} \leftarrow c_{j}^{\prime}+c_{i}^{t}$. If one of the elements, say $c_{i}^{\prime}=0$, then $c_{j}^{\prime}$ has a probability of 0.5 to carry out the above shifting operation. If both elements are zero, the operation of this element pair is aborted.

3) Operational Procedures: The operational procedures of CAA are given in Algorithm 1. $\mathcal{L}$ is the Tabu list that contains the solutions visited in recent past and its maximum length is $L$. Initially, $\mathcal{L}$ only contains one solution candidate, $\boldsymbol{c}_{s}^{\prime}$, which is randomly generated with equality constraint (17) preserved. $c_{s}^{\prime}$ is also referred as the current local best and considered as global best (denoted by $\boldsymbol{c}_{*}^{\prime}$ ) (line 2). In each iteration, a set of neighbors of $\boldsymbol{c}_{s}^{\prime}, R\left(\boldsymbol{c}_{\boldsymbol{s}}^{\prime}\right)$, is obtained (line 4) via Pairing and Shifting operations. If the best non-Tabu neighbor in $R\left(\boldsymbol{c}_{s}^{\prime}\right)$ outperforms $\boldsymbol{c}_{s}^{\prime}$ in terms of the corresponding optimal EV flow distribution solution $\hat{q}_{I}, \boldsymbol{c}_{s}^{\prime}$ is updated (line 6-7). Similarly, $\boldsymbol{c}_{*}^{\prime}$ is also updated if $\boldsymbol{c}_{s}^{\prime}$ is better (line 9-10). Lastly, this solution is added into the Tabu list $\mathcal{L}$ (line 11-13). Such a searching cycle repeats until the number of iterations reaches $E$.

The outputs of the algorithm, i.e. $\boldsymbol{c}^{*}$ and $\boldsymbol{c}_{*}^{\prime}$, are the resultant charger allocation and the corresponding placement strategy that benefit the optimal EV flow distribution under stochastic traffic flow $\delta$ the most.

Remark 1. The most time-consuming procedure of CAA is the evaluation of non-Tabu neighbors, where the corresponding problem (7) instances are solved by SQP. As a result, the computational cost of CAA would depend on that of SQP. In each CAA iteration, there is at most $\left|R\left(\boldsymbol{c}_{\boldsymbol{s}}^{\prime}\right)\right|$ non-Tabu neighbors generated. Thus, the total number of SQP execution in $C A A$ is at most $E \times\left|R\left(\boldsymbol{c}_{s}^{\prime}\right)\right|$. In Sect. IV-B2, the running time of $S Q P$ will be further studied.

\section{Simulation Results and Discussions}

\section{A. Dataset and Parameter Settings}

To demonstrate the effectiveness of the proposed problem framework and solution algorithm, the eastern Massachusetts highway network [30] shown in Fig. 2 is employed.

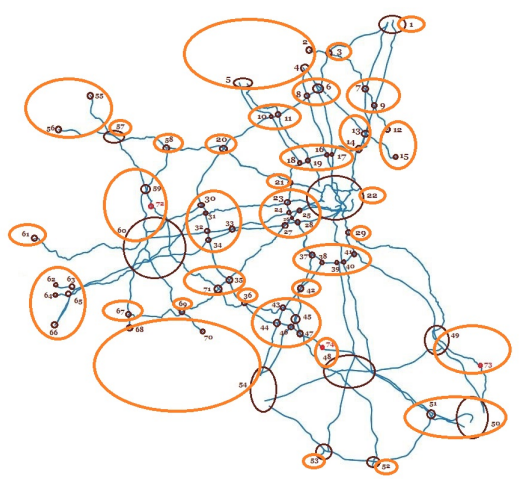

Figure 2: The eastern Massachusetts highway subnetwork. Each node of the network corresponds to a "zone", which is an area integrating several locations.

The highway network is modeled as a directed and weighted graph $\mathcal{G}=(\mathcal{V}, \mathcal{E}, \mathcal{A})$ with $|\mathcal{V}|=74$ and $|\mathcal{E}|=258$. The distances (in $\mathrm{km}$ ), free-flow travel time (in hours) and flow capacity of each edge are respectively employed for $d_{i j}, \tau_{i j}^{0}$ and $q_{i j}$ in $\mathcal{A}$. The charging stations $\mathcal{V}_{c}$ are sited at nodes with 
the highest betweenness centrality [31], and for any unreachable pairs $(m \mapsto n), \lambda(m, n)=\lambda$ is assumed. Table I lists the general settings of other parameters for the simulations. It is remarked that these settings are for illustrative purpose and they can be fully adjustable according to practical situations. All the simulations are implemented with MATLAB on a PC platform with Intel Core i7-6700H CPU @ 2.60GHz and 8 GB of RAM. In particular, the SQP method is implemented in "fmincon" function of optimization toolbox [32].

Table I: Parameter Settings

\begin{tabular}{ll}
\hline \hline Parameters & Settings \\
\hline Number of charging stations $\left(\left|\mathcal{V}_{c}\right|\right)$ & 10 \\
Initial number of chargers $\left(c_{k}^{0}, \forall s_{k} \in \mathcal{V}_{c}\right)$ & 3 \\
Utilization of road capacity $\left(\Delta_{i j}, \forall(i, j) \in \mathcal{E}\right)$ & $\mathcal{N}(0.5,0.1)$ \\
EV's re-charge time $\left(F_{c} \sim \mathcal{N}\left(\mu_{c}, \sigma_{c}^{2}\right)\right)$ & $\mathcal{N}(0.5,0.13)$ \\
$F_{c}$ 's truncation interval & {$[0.3,0.7]$} \\
Retained charging resources $(\epsilon)$ & $10 \%$ \\
Number of samples in SAA $(I)$ & 20 \\
Maximum size of Tabu list $(L)$ & 5 \\
Maximum iteration of CAA $(E)$ & 100 \\
Number of neighbors generated for $\boldsymbol{c}_{\boldsymbol{s}}^{\prime}\left(\left|R\left(\boldsymbol{c}_{\boldsymbol{s}}^{\prime}\right)\right|\right)$ & 10
\end{tabular}

\section{B. Algorithmic Performance Analysis}

1) Quality of SAA Solution: The quality of SAA solution with respect to the solution of original two-stage problem (4) is firstly evaluated. Assuming the optimal objective value for problem (4) is $q^{*}$, then $\hat{q}_{I}\left(\boldsymbol{c}^{*}\right) \geq q^{*}$ must hold, where $\boldsymbol{c}^{*}$ is obtained by solving the corresponding SAA problem (16). The quality of $c^{*}$ can thus be measured by the optimality gap, which is defined as

$$
P\left(c^{*}\right):=\hat{q}_{I}\left(c^{*}\right)-q^{*}
$$

In [23], a statistical procedure is outlined to estimate $P\left(c^{*}\right)$ which is given by

$$
\hat{P}\left(c^{*}\right):=U B\left(c^{*}\right)-L B
$$

where $U B\left(c^{*}\right)$ and $L B$ are the confidence upper-bound and lower-bound of $\hat{q}_{I}\left(c^{*}\right)$, respectively.

Let the $\mathrm{EV}$ flow rate $\lambda=2(\mathrm{EV}$ s/hour) at each unreachable source-destination pair and the total number of chargers for allocation $U=30$, the quality of $c^{*}$ with respect to EV's driving range $d_{r}$ is presented in Table II. Note that three ranges, i.e. $180,175,170(\mathrm{~km})$ are examined because such variations with respect to $d_{r}$ lead to different sets of unreachable source-destination pairs, covering various scenarios for the second-stage optimization problem (7).

Table II shows that the magnitude of the estimated optimality gaps $\hat{P}\left(c^{*}\right)$ are less than 0.02 , which means the number of samples in SAA $(I=20)$ is good enough to handle the uncertainty induced by stochastic traffic flow $\delta$.
Table II: Estimation of quality of $c^{*}$ for different $d_{r}$

\begin{tabular}{llll}
\hline \hline Ranges $\left(d_{r}\right)$ & $U B\left(\boldsymbol{c}^{*}\right)$ & $L B$ & $\hat{P}\left(\boldsymbol{c}^{*}\right)$ \\
\hline $180(\mathrm{~km})$ & 2.8419 & 2.8304 & 0.0115 \\
$175(\mathrm{~km})$ & 2.8374 & 2.8339 & 0.0036 \\
$170(\mathrm{~km})$ & 2.9985 & 2.9815 & 0.0170 \\
\hline
\end{tabular}

2) Running Time of $S Q P$ : Fig. 3 depicts the running times for SQP in solving problem (7) based on five different values of $d_{r}$. Since the running time may vary, 30 problem (7) instances are solved for each $d_{r}$. The results show that the smaller $d_{r}$ is, the more time SQP takes to solve the problem. It is because, given a smaller $d_{r}$, the number of decision variables $\lambda_{i j}^{(m n)}$, i.e. rates of EV flows assigned at "tree branches", would increase together with the number of unreachable source-destination pairs. However, one SQP execution only takes a few seconds in all considered cases, and thus is acceptable for practical applications.

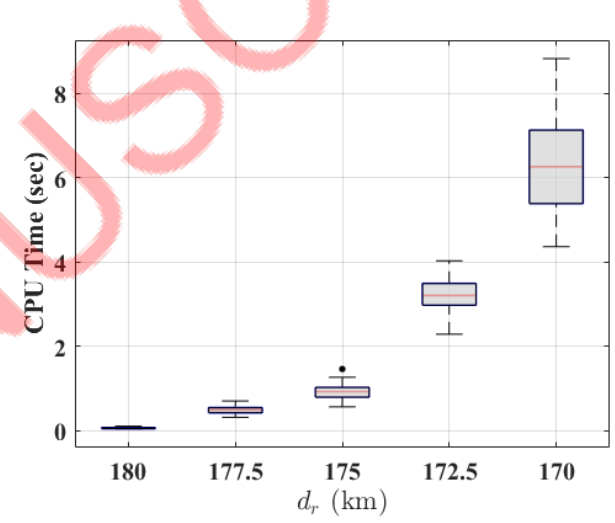

Figure 3: Running time of SQP under different $d_{r}$.

3) Effectiveness of $S Q P$ : In [3], a flow distribution algorithm (FDA) is suggested to assign EV flows to feasible paths with deterministic traffic conditions. FDA is based on genetic algorithm [33] that is composed of binary tournament selection, simulated binary crossover and polynomial mutation. It can also be applied to solve problem (7). Let $d_{r}=175(\mathrm{~km}), U=30$, and $\mu_{i j}=0.5$, then for each $\lambda \in\{\lambda \in \mathbb{N}: 1 \leq \lambda \leq 6\}, 50$ sets of problem (7) are formulated under randomly generated charger allocations. These problem sets are respectively solved by both SQP and FDA for comparison. Since the uncertainty induced by $\boldsymbol{\delta}$ would also affect the decision-making in (7), the same randomly generated $I$ samples of $\boldsymbol{\delta}$ are adopted for each charger allocation. The quality of a flow distribution solution under any charger allocation $\boldsymbol{c}$ can thus be measured by averaging $H^{*}(\boldsymbol{c}, \boldsymbol{\delta})$ over $I$ traffic samples. Therefore, $\hat{q}_{I}(\boldsymbol{c})$ essentially becomes the algorithmic performance metric instead of $H(\boldsymbol{c}, \boldsymbol{\delta})$.

The performance improvements of SQP over FDA in terms $\hat{q}_{I}(\boldsymbol{c})$ and CPU time consumption, are presented in Fig. 4 (a) and (b), respectively. It is clearly observed that SQP not only obtains solutions with more than $15 \%$ less $\hat{q}_{I}(\boldsymbol{c})$ (except for $\lambda=1$ ), but also saves at least $70 \%$ of the CPU time. As proved in Theorem 1, problem (7) is convex and applying SQP as a 
solver would guarantee to find the optimal EV flow rates $\Lambda^{*}$. By contrast, heuristic algorithms like FDA do not have any performance guarantee. Therefore, SQP outperforms FDA in all cases.

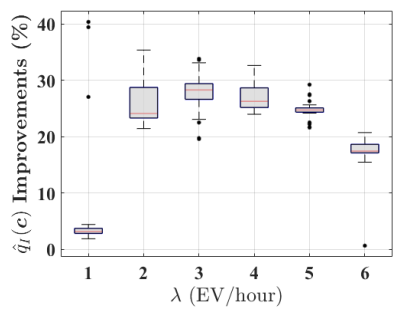

(a)

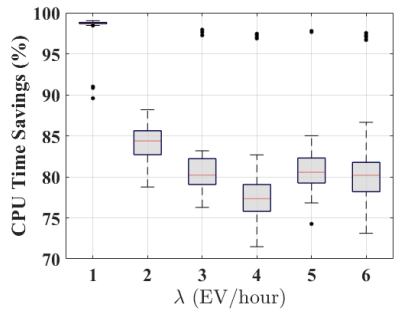

(b)
Figure 4: Performance improvement of SQP against FDA (a) solution quality (b) CPU time consumption.

4) Effectiveness of CAA: [3] also suggests two charger allocation schemes, namely proportional allocation (PA) and uniform allocation (UA). PA assigns more chargers to charging nodes with higher betweenness, while UA assigns equal number of chargers to each of charging stations. Let SQPPro/SQP-Uni denote the algorithms that solve problem (16) with PA/UA allocation and SQP. For comparison purpose, CAA, SQP-Pro and SQP-Uni are applied to solve the same sets of problem (16). Fig. 5 shows the specific parameter settings and simulation results.

Fig. 5 (a) clearly demonstrates the out-performance of CAA over SQP-Pro and SQP-Uni by achieving the smallest expected total journey time of $\mathrm{EV} \hat{q}_{I}$. However, it is noticed that the improvement is less significant when the arrival rate of EV flow at each source node is small, e.g. $\lambda=1$. To better explore the reason, the composition of $\hat{q}_{I}$ is obtained. In Fig. 6 (a), EV's (expected) traveling delay on the road $\hat{q}_{I}^{(T)}$ and the total delay at the charging stations $\hat{q}_{I}^{(W)}$ (including both queuing and charging time), as given in (2), are obtained against $\lambda$ using SQP. It shows that $\hat{q}_{I}^{(T)}$ remains similar but $\hat{q}_{I}^{(W)}$ decreases noticeably with fewer EVs demanding en-route recharging, i.e. smaller $\lambda$. With a small $\lambda$, the charging resource would be relatively "sufficient" and EVs experience very little (or even no) queuing delay at the charging stations. As a result, the allocation of additional chargers becomes less critical and applying CAA can no longer achieve a significant reduction in $\hat{q}_{I}^{(W)}$, as shown in Fig. 5 (a). Also, from Fig. 5 (a), SQP-Pro performs better than SQP-Uni, which is consistent with the results given in [3].

Fig. 5 (b) presents the cases where the number of chargers for allocation $(U)$ is increased from 30 to 50. As compared to Fig. 5 (a), $\hat{q}_{I}$ of all the algorithms drops. Although CAA is still the best, the induced improvement becomes less. Charging resource sufficiency, as a direct implication of increasing $U$, is again the main reason. This is also confirmed by comparing Fig. 6 (a) and (b), showing that decreasing $\lambda$ and increasing $U$ have similar impacts on $\hat{q}_{I}$. Therefore, CAA's solutions hardly benefit from the more-than-sufficient charging resources for $1 \leq \lambda \leq 4$, as shown in Fig. 5 (b). However, when the charging resource becomes relatively "insufficient" by increasing $\lambda$ to
5 or 6 (EV/hour), $\hat{q}_{I}$ improvements provided by CAA become noticeable.

In Fig. 5 (c), EV's driving range $d_{r}$ is set to $180(\mathrm{~km})$ rather than $175(\mathrm{~km})$, and again, the CAA's out-performance becomes less significant. Extending EV's range would reduce the number of unreachable source-destination pairs. Consequently, service load at charging stations drops, which turns out to be the same case as discussed following Fig. 5 (a).

In Fig. $5(\mathrm{~d})$, the mean utilization of edge $(i, j)$ capacity $\mu_{i j}$ is decreased to 0.3 . Based on (1), less traffic volume shall lead to less travel delay along the edges and thus smaller $\hat{q}_{I}$. The decrease in $\hat{q}_{I}$ (as compared to Fig. 5 (a)) is marked in red in Fig. 5 (d). As shown, such a decrease is not substantial for all the tested cases, and CAA remains dominant.

In summary, CAA can solve problem (16) effectively under all the testing scenarios. Its out-performance over the other approaches is especially significant when the charging resource is tight.
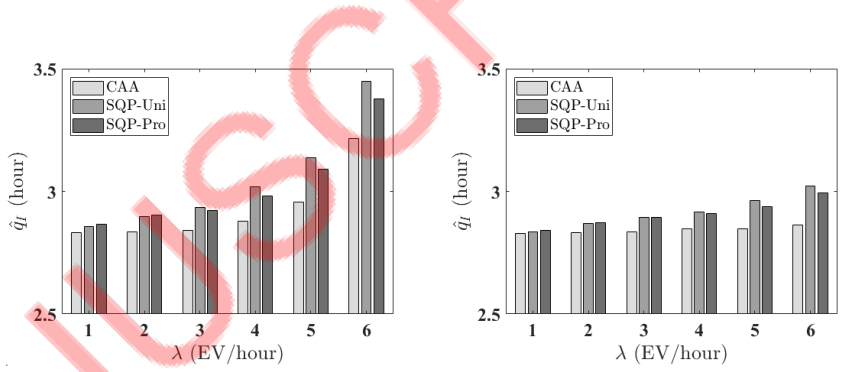

(a) $d_{r}=175(\mathrm{~km}), U=30$, and $\mu_{i j}=0.5$

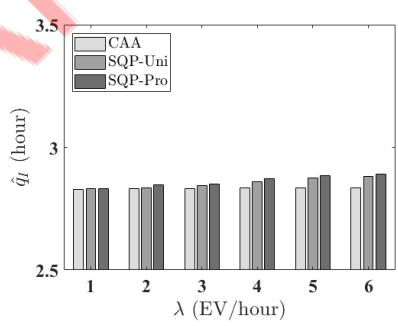

(b) $d_{r}=175(\mathrm{~km}), U=50$, and $\mu_{i j}=0.5$

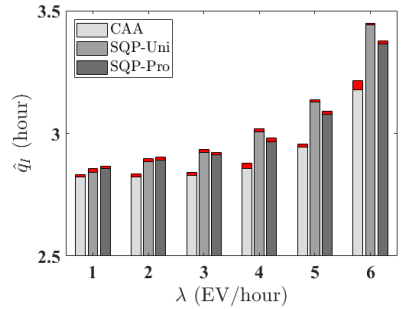

(c) $d_{r}=180(\mathrm{~km}), U=30$, and (d) $d_{r}=175(\mathrm{~km}), U=30$, and $\mu_{i j}=0.5$ $\mu_{i j}=0.3$

Figure 5: Performance evaluation of CAA.

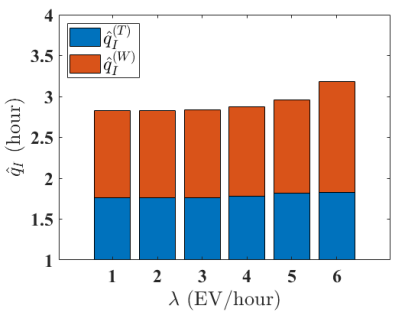

(a) $U=30$

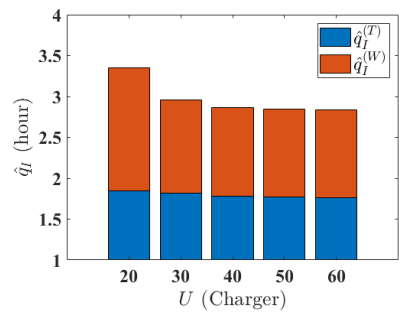

(b) $\lambda=5(\mathrm{EV} / \mathrm{hour})$
Figure 6: Composition of $\hat{q}_{I}$, where $d_{r}=175(\mathrm{~km})$.

\section{Impacts of Parameter Variations}

The results presented in Fig. 5 not only verify the effectiveness of CAA, but also suggest that the parameter setting 
would affect the solutions of problem (16). In this section, the impacts of major parameters, including charging resource sufficiency $(U)$, driving range $\left(d_{r}\right)$ and stochastic traffic flow $\left(\Delta_{i j}\right)$, are extensively studied.

1) Impact of Charging Resources Sufficiency: Fig. 5 (a)-(c) suggest that the expected total journey time of the EV can be greatly reduced when the charging resource is sufficient for EVs to re-charge without queuing. A natural question arose here is how to define "sufficient" quantitatively. In other words, is there a lower bound for the number of chargers to allocate $(U)$, such that the EV flow with bounded arrival rate can always re-charge without queuing?

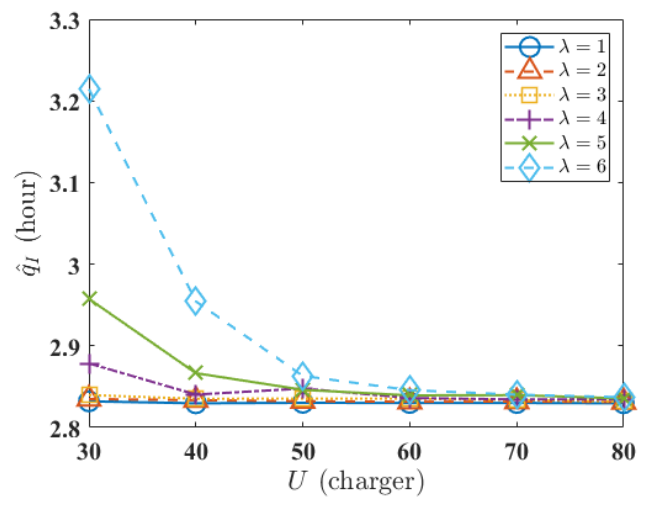

Figure 7: The expected total journey time of EV $\left(\hat{q}_{I}\right)$ versus the number of chargers for allocation $(U)$, with $d_{r}=175(\mathrm{~km})$ and $\mu_{i j}=0.5$.

To answer this question, the expected total journey time of $\mathrm{EV}\left(\hat{q}_{I}\right)$ is derived with respect to the number of chargers for allocation $(U)$, under various arrival rate of EV flows. The specific settings and results are presented in Fig. 7. $U$ starts with 30 which is the least number of chargers that satisfies the inequality (19) under the current settings, and it is denoted by $U^{e}$ hereafter. Fig. 7 shows that, given any $\lambda, \hat{q}_{I}$ decreases when $U$ increases. In particular, when $U \geq 60$, the drop of $\hat{q}_{I}$ is less than a threshold $\xi=0.01$. It implies the elimination of queuing and $\hat{q}_{I}$ is only further improved with a shortened (expected) re-charging time. 60 (chargers) can thus be considered as an estimator of the lower bound of $U$ with the EV flow rate $\lambda \leq 6$ (EV/hour), and symbolized by $U_{\xi=0.01}^{b}$. It's also noted that a more precise lower bound of $U$ is possible by increasing $U$ with a smaller step size.

In practice, the rate of EV flows in a road network is highly dependent to the market share of EV. With the market share of $\mathrm{EV}$ at a future point being statistically predictable, this lower bound for chargers would be a very useful guideline, to prevent over-investment of charging resources.

2) Impact of Driving Range: Fig. 5 (c) demonstrate that the extension of EV's driving range $d_{r}$ helps to reduce the charging load, and the number of chargers that is required for a quality re-charge service can be reduced accordingly.

In Fig. 8 (a)-(d), EV's expected total journey times $\left(\hat{q}_{I}\right)$ are derived with respect to the number of chargers for allocation $(U)$, under different $d_{r}$. Let $\xi=0.01$, the least number of chargers $U^{e}$ and the lower bound $U_{\xi=0.01}^{b}$ for different $d_{r}$ can

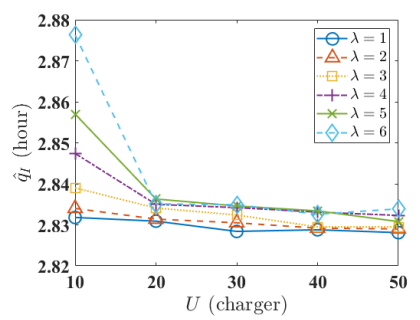

(a) $d_{r}=180(\mathrm{~km})$

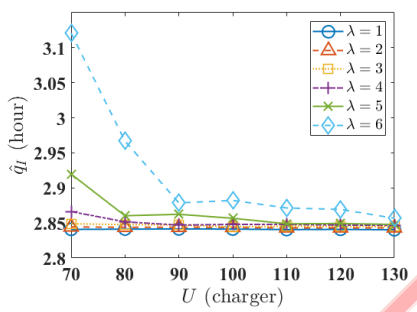

(c) $d_{r}=172.5(\mathrm{~km})$

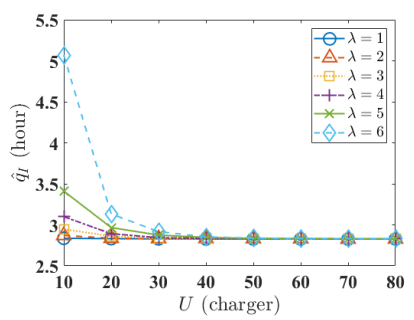

(b) $d_{r}=177.5(\mathrm{~km})$

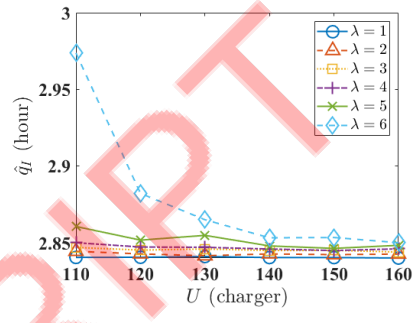

(d) $d_{r}=170(\mathrm{~km})$

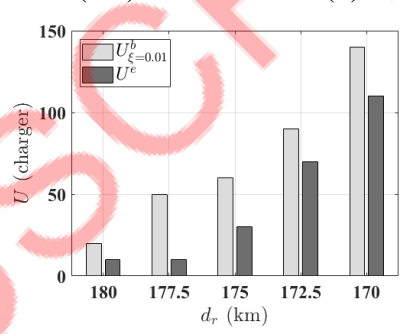

(e) $U_{\xi=0.01}^{b}$ and $U^{e}$ against $d_{r}$

Figure 8: Charging resource sufficiency under different driving range of $\mathrm{EV}$, with $\mu_{i j}=0.5$.

thus be obtained as shown in Fig. 8 (e). The results indicate that both $U^{e}$ and $U_{\xi=0.01}^{b}$ decrease with the increase of $d_{r}$. Therefore, the allocation planning of charging resources would also be benefited from the advance of EV's battery technology.

3) Impact of Stochastic Traffic Flow: Fig. 5 (d) has implied that the traffic flow on edge $(i, j)$ characterized by $\Delta_{i j}$ may not have substantial impact on the solutions of problem (16) (obtained by CAA). For further investigation, EV's expected total journey times $\left(\hat{q}_{I}\right)$ are acquired with respect to EV flow rate at each source $(\lambda)$ under different $\Delta_{i j}$. The settings and the results are given in Fig. 9. In Fig. 9 (b), the number of chargers for allocation $(U)$ is increased to 50 so as to study cases where the queuing delay is largely obviated with given sufficient charging resources.

The comparison between Fig. 9 (a) and Fig. 9 (b) clearly shows that the queuing delay caused by excessive re-charge activities (for $\lambda=5,6$ in Fig. 9 (a)) leads to a serious degradation in $\hat{q}_{I}$, while the travel delay induced by the extra vehicle traffic is little (see Fig. 9 (b)). In conclusion, comparing to the variations in traffic conditions, the sufficiency of charging resources plays a more critical role in the distribution of $\mathrm{EV}$ flows.

\section{Conclusion}

In this paper, a two-stage stochastic programming model is considered to coordinate the allocation of chargers and the 


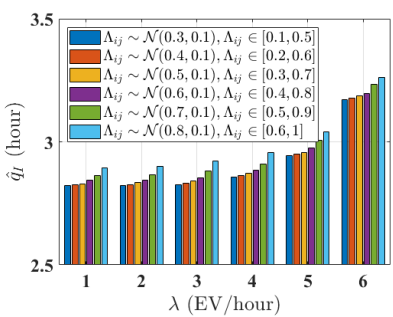

(a) $U=30$

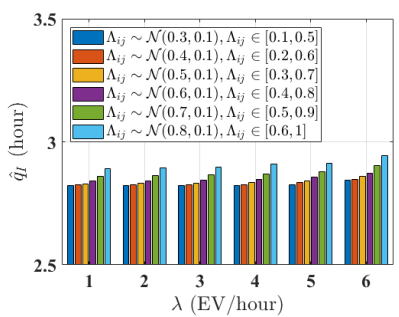

(b) $U=50$
Figure 9: Impact of stochastic traffic flow, with $d_{r}=175(\mathrm{~km})$.

distribution of EV flows under stochastic traffic conditions. The EV's expected total journey time is minimized jointly by the two-stage decisions. Based on the SAA method, a feasible deterministic equivalent of the original problem is derived. Furthermore, the EV flow distribution problem is proved to be convex. Therefore, the two-stage optimization problem can be effectively solved by a hybrid approach, which incorporates SQP and a Tabu-based heuristic, so that a high-quality charger allocation and optimal EV flow distribution policy are obtained. Numerical results confirm the quality of SAA estimator as well as the out-performance of the hybrid approach over existing ones. Moreover, the proposed formulation designates the sufficiency in terms of charging resources by statistically estimating a lower bound for the number of chargers to allocate, which in practice helps to prevent over-allocation of charging resources. The simulation results also reveal that the sufficiency of charging resources is critical for EV's driving experience, because it has greater impact on traveling delay comparing to the variation in traffic conditions. In the future, this work would be extended to consider bidirectional chargers for the implementation of vehicle-to-grid (V2G) services [34], [35].

\section{REFERENCES}

[1] International Energy Agency (IEA), "Global EV Outlook 2019." [Online]. Available: www.iea.org/publications/reports/globalevoutlook2019/

[2] Electric Vehicles Initiative (EVI), “Ev30@30 Campaign.” [Online]. Available: https://www.iea.org/topics/transport/evi/

[3] X. Bi, W. K. S. Tang, Z. Han, and J. Zhou, "Distributing electric vehicles to the right charging queues," in Proc. IEEE Int. Symp. Circuits Syst. IEEE, 2019, pp. 1-5.

[4] B. Alinia, M. H. Hajiesmaili, and N. Crespi, "Online EV charging scheduling with on-arrival commitment," IEEE Trans. Intell. Transp. Syst., vol. 20, no. 12, pp. 4524-4537, 2019.

[5] C. De Cauwer, W. Verbeke, J. Van Mierlo, and T. Coosemans, "A model for range estimation and energy-efficient routing of electric vehicles in real-world conditions," IEEE Trans. Intell. Transp. Syst., vol. 21, no. 7, pp. 2787-2800, 2019.

[6] M. Latifi, A. Khalili, A. Rastegarnia, and S. Sanei, "A Bayesian realtime electric vehicle charging strategy for mitigating renewable energy fluctuations," IEEE Trans. Ind. Inf., vol. 15, no. 5, pp. 2555-2568, 2018.

[7] X. Bi and W. K. S. Tang, "Logistical planning for electric vehicles under time-dependent stochastic traffic," IEEE Trans. Intell. Transp. Syst, vol. 20, no. 10, pp. 3771-3781, 2019.

[8] Z. Yang, T. Guo, P. You, Y. Hou, and S. J. Qin, "Distributed approach for temporal-spatial charging coordination of plug-in electric taxi fleet," IEEE Trans. Ind. Inf., vol. 15, no. 6, pp. 3185-3195, 2018.

[9] A. Bourass, S. Cherkaoui, and L. Khoukhi, "Secure optimal itinerary planning for electric vehicles in the smart grid," IEEE Trans. Ind. Inf., vol. 13, no. 6, pp. 3236-3245, 2017.
[10] H. Yang, Y. Deng, J. Qiu, M. Li, M. Lai, and Z. Y. Dong, "Electric vehicle route selection and charging navigation strategy based on crowd sensing," IEEE Trans. Ind. Inf., vol. 13, no. 5, pp. 2214-2226, 2017.

[11] M. Li, X. Wu, Z. Zhang, G. Yu, Y. Wang, and W. Ma, "A wireless charging facilities deployment problem considering optimal traffic delay and energy consumption on signalized arterial," IEEE Trans. Intell. Transp. Syst., vol. 20, no. 12, pp. 4427-4438, 2019.

[12] W. Infante, J. Ma, X. Han, and A. Liebman, "Optimal recourse strategy for battery swapping stations considering electric vehicle uncertainty," IEEE Trans. Intell. Transp. Syst., vol. 21, no. 4, pp. 1369-1379, 2019.

[13] E. Kianmehr, S. Nikkhah, V. Vahidinasab, D. Giaouris, and P. C. Taylor, "A resilience-based architecture for joint distributed energy resources allocation and hourly network reconfiguration," IEEE Trans. Ind. Inf., vol. 15 , no. 10 , pp. 5444-5455, 2019.

[14] T. Zeng, H. Zhang, and S. Moura, "Solving overstay and stochasticity in PEV charging station planning with real data," IEEE Trans. Ind. Inf., vol. 16, no. 5, pp. 3504-3514, 2019.

[15] Q. Cui, Y. Weng, and C.-W. Tan, "Electric vehicle charging station placement method for urban areas," IEEE Trans. Smart. Grid., 2019.

[16] Y. Xiong, J. Gan, B. An, C. Miao, and A. L. Bazzan, "Optimal electric vehicle fast charging station placement based on game theoretical framework," IEEE Trans. Intell. Transp. Syst., vol. 19, no. 8, pp. 2493 2504, 2018.

[17] S. Wang, Z. Y. Dong, F. Luo, K. Meng, and Y. Zhang, "Stochastic collaborative planning of electric vehicle charging stations and power distribution system," IEEE Trans. Ind. Inf., vol. 14, no. 1, pp. 321-331, 2017.

[18] G. Wang, X. Zhang, H. Wang, J.-C. Peng, H. Jiang, Y. Liu, C. Wu, Z. Xu, and W. Liu, "Robust planning of electric vehicle charging facilities with an advanced evaluation method," IEEE Trans. Ind. Inf., vol. 14, no. 3, pp. 866-876, 2017.

[19] Bureau of Public Roads, Traffic Assignment Manual. Urban Planning Division: Office of Planning, 1964.

[20] M. Zukerman, "Introduction to queueing theory and stochastic teletraffic models," arXiv preprint arXiv:1307.2968, 2013.

[21] ChargeHub, "Electric Vehicle Charging Guide," 2019. [Online]. Available: https://chargehub.com/en/electric-car-charging-guide.html

[22] A. Lee and P. Longton, "Queueing processes associated with airline passenger check-in," J. Oper. Res. Soc., vol. 10, no. 1, pp. 56-71, 1959.

[23] A. Shapiro and A. Philpott, "A tutorial on stochastic programming," Manuscript. Available at www2.isye.gatech. edu/ ashapiro/publications. html, vol. 17, 2007.

[24] X. Bi and W. K. Tang, "Flow distribution for electric vehicles under nodal-centrality-based resource allocation," IEEE Trans. Circuits Syst. I Regul. Pap., vol. 67, no. 4, pp. 1309-1318, 2019.

[25] S. Boyd and L. Vandenberghe, Convex Optimization. Cambridge University press, 2004.

[26] R. A. Horn and C. R. Johnson, Matrix Analysis. Cambridge University press, 2012

[27] H. L. Lee and M. A. Cohen, "A note on the convexity of performance measures of $\mathrm{m} / \mathrm{m} / \mathrm{c}$ queueing systems," J. Appl. Probab., vol. 20, no. 4, pp. 920-923, 1983.

[28] P. T. Boggs and J. W. Tolle, "Sequential quadratic programming," Acta Numer, vol. 4, no. 1, pp. 1-51, 1995.

[29] F. Glover and M. Laguna, "Tabu search," in Handbook of combinatorial optimization. Springer, 1998, pp. 2093-2229.

[30] "Github - Transportation Networks for Research," https://github.com/b stabler/TransportationNetworks, (Accessed on 10/11/2018).

[31] G. Chen, X. Wang, and X. Li, Fundamentals of complex networks: models, structures and dynamics. John Wiley \& Sons, 2014.

[32] "MATLAB Optimization Toolbox: Fmincon," Matlab R2018b, the MathWorks, Natick, MA, USA

[33] K. F. Man, K. S. Tang, and S. Kwong, Genetic Algorithms: Concepts and Designs. Springer Verlag, London, 1999.

[34] K. M. Tan, V. K. Ramachandaramurthy, and J. Y. Yong, "Integration of electric vehicles in smart grid: A review on vehicle to grid technologies and optimization techniques," Renewable Sustainable Energy Rev., vol. 53, pp. 720-732, 2016.

[35] F. Mwasilu, J. J. Justo, E.-K. Kim, T. D. Do, and J.-W. Jung, "Electric vehicles and smart grid interaction: A review on vehicle to grid and renewable energy sources integration," Renewable Sustainable Energy Rev., vol. 34, pp. 501-516, 2014. 